D.O.I.: $10.3895 / \mathrm{S} 1808-04482007000400011$

\title{
MODELO DE DECISÃO PARA SELEC̃̃̃ DE SISTEMAS DE INFORMAÇÃO BASEADO EM DECISÃO MULTICRITÉRIO E PROGRAMAÇÃO INTEIRA 0-1
}

\section{DECISION MODEL TO INFORMATION SYSTEMS SELECTION BASED ON MULTICRITERIA DECISION AND INTEGER PROGRAMMING 0-1}

\author{
Yuri Lopes ${ }^{1}$; Ana Paula Cabral Seixas Costa ${ }^{2}$ \\ ${ }^{1}$ Federal University of Pernambuco - UFPE - Recife - Brasil yurilopes@ufpe.br \\ ${ }^{2}$ Federal University of Pernambuco - UFPE - Recife - Brasil apcabral@ufpe.br
}

\begin{abstract}
Resumo
Atuar num ambiente de competição requer das empresas a utilização eficiente de seus recursos financeiros, a fim de trazer vantagem competitiva, impulsionando o negócio. Para que as potenciais vantagens da tecnologia da informação (TI) e dos sistemas de informação (SI) sejam alcançadas é primordial uma gestão eficiente destes recursos, sobretudo nas decisões de investimento em tecnologia da informação. A TI não pode ser avaliada unicamente pelos ganhos financeiros, mas também pelas demais contribuições que ela traz aos vários aspectos do negócio, o que caracteriza o problema de investimentos em TI/SI como um problema de decisão multicritério. Neste trabalho, é proposto um modelo baseado no método PROMETHEE $V$, uma combinação entre o método de decisão multicritério PROMETHEE II e programação inteira 0-1, envolvendo diferentes tipos de atributos e restrições presentes no problema.
\end{abstract}

Palavras-chave: seleção de investimentos, tecnologia da informação, apoio a decisão.

\section{Introdução}

Atuar num ambiente de competição requer das empresas a utilização eficiente de seus recursos financeiros, a fim de trazer vantagem competitiva, impulsionando o negócio. A aplicação desses recursos em projetos, máquinas ou recursos humanos deve atender as circunstanciais necessidades organização, assim como vislumbrar boas oportunidades no médio e longo prazo. A utilização de ferramentas de tecnologia da informação e de sistemas de informação (TI/SI) que impulsionem o negócio é primordial à sobrevivência nos mercados competitivos; tais ferramentas aumentam a eficiência operacional, a capacidade de resolução de problemas, além de facilitar o processo de tomada de decisão dentro da organização. 
Para que as potenciais vantagens da TI/SI sejam alcançadas é primordial uma gestão eficiente destes recursos, sobretudo nas decisões de investimento em tecnologia da informação. Essas decisões são estratégicas para a empresa, direcionam suas atividades em médio e longo prazo; um equívoco na política de TI da empresa pode significar prejuízos significativos, ou até a falência da organização. É um consenso na literatura de SI que as organizações precisam mais do que nunca de processos que permitam decidir pelos SI mais apropriados, o que significa a existência de um alinhamento entre a estratégia do negócio e a estratégia de SI (ALBUQUERQUE, 2006).

Este artigo discute a problemática de investimentos em tecnologia da informação (TI), sobretudo quanto à priorização de potenciais aplicações de TI/SI, que compõem o portfolio de investimentos deste setor da empresa.

$\mathrm{O}$ artigo está dividido da seguinte forma: o item 2 abordará as características do problema de priorização de investimentos em TI. Um modelo de decisão combinando um método multicritério de apoio a decisão com programação inteira 0-1 será proposto (item 3) e aplicado (item 4) a uma empresa estadual de fomento em TI, localizada no Nordeste do Brasil. Por último, serão apresentadas as conclusões deste trabalho.

\section{Priorização de investimentos em TI/SI}

Além da necessidade de transportar a estratégia do negócio para aplicações de SI, as organizações deparam-se com o problema de depois de identificados os SI mais apropriados e construído o portfólio de projetos de SI, definir a ordem de implementação desses projetos diante da limitação de recursos (ALBUQUERQUE, 2006).

Grande parte dos trabalhos desenvolvidos na área de priorização de investimentos procura obter o maior retorno financeiro possível diante das opções de investimentos da empresa. No contexto de SI/TI, a procura pelo retorno financeiro dos projetos é difícil e bastante subjetiva. Transformar melhorias operacionais e eficiência administrativa, por exemplo, numa escala monetária é complicado e, muitas vezes, inviável. A decisão de investir em Sistemas de Informação (SI) e em Tecnologia de Informação tem sido alvo de inúmeras discussões, principalmente pela dificuldade de quantificar os benefícios advindos desse investimento.

Grandes investimentos em estrutura de hardware e software não se traduzem, necessariamente, em reais benefícios para a empresa. É preciso identificar quais são e onde devem ser aplicados os SI's e as ferramentas de TI. A integração entre os SI's utilizados é de grande importância no alcance dos objetivos estratégicos da empresa; não adianta construir "ilhas" de prosperidade na organização se esses avanços não puderem ser compartilhados entre os demais 
setores e ajudá-los nas suas tarefas (LOPES \& COSTA, 2006). Deve existir, portanto, na gestão empresarial, uma análise formal para tomada de decisão quanto à priorização desses investimentos.

Genericamente, a priorização de sistemas de informação é realizada tendo como base dois aspectos: a análise das características (atributos) dos sistemas de informação e a análise custobenefício associada aos sistemas (AHITUV \& NEUMANN, 1983). É preciso definir a ordem de implementação desses sistemas diante da limitação de recursos, o que representa efetivamente a decisão de investimento em SI/TI. Vários são os métodos utilizados no problema de priorização de SI's: árvores de decisão, técnica Delphi, programação inteira, programação dinâmica, programação quadrática, programação linear,etc.

Segundo Schwarz \& Hirschheim (2003), as organizações utilizam uma métrica multifacial de sucesso, não limitada somente a benefícios financeiros da TI, mas como a TI tem sido percebida. A TI/SI não pode ser avaliada unicamente pelos ganhos financeiros, mas também pelas demais contribuições que ela traz aos vários aspectos do negócio. Dessa forma, a priorização de investimentos em SI/TI caracteriza-se como um problema de decisão multicritério.

\section{Modelo proposto}

A construção do modelo de decisão de priorização de investimentos em TI/SI baseria-se numa combinação entre um método de decisão multicritério e programação inteira (0-1), o PROMETHEE V (BRANS et al, 1992). Na primeira parte, será utilizado o método PROMETHEE II, a fim de tratar o problema em seus diferentes aspectos, considerando vários critérios de avaliação das alternativas de investimento. A partir desta avaliação multicritério, um modelo de programação inteira (0-1) buscará maximizar o retorno do portfolio de investimento observando algumas restrições, relacionadas com os recursos disponíveis e com aspectos gerenciais da função de TI.

O Apoio Multicritério a Decisão (AMD) consiste em um conjunto de métodos e técnicas para auxiliar ou apoiar pessoas e organizações a tomarem decisões, sob a influência de uma multiplicidade de critérios. Tem como princípio buscar o estabelecimento de uma relação de preferências (subjetivas) entre as alternativas que estão sendo avaliadas sob a influencia de vários critérios (ALMEIDA \& COSTA, 2003).

Segundo Bouyssou (1990) apud Mello et al(2005), uma abordagem multicritério apresenta as seguintes vantagens:

- torna viável a construção de uma base para o diálogo entre analistas e decisores, que fazem uso de diversos pontos de vista comuns;

- provê facilidade em incorporar incertezas aos dados sobre cada ponto de vista; 
- permite encarar cada alternativa como um compromisso entre objetivos em conflito. Este argumento destaca o fato de que raramente será encontrada uma situação em que exista uma alternativa superior às restantes sobre todos os pontos de vista.

Segundo Vincke (1992), um problema de decisão multicritério é uma situação em que, sendo definido um conjunto de ações A, e uma família de critérios F, deseja-se:

a) Determinar um subconjunto de ações considerada a melhor em relação a F (problema de escolha);

b) Dividir A em subconjuntos de acordo com algumas normas (problema de classificação);

c) Ordenar as ações de A da melhor para a pior (problema de ordenação);

Tradicionalmente, pode-se classificar os métodos multicritério em compensatórios e não compensatórios. O primeiro grupo, mais conhecido como Escola Americana, assume que o decisor é capaz de explicitar suas preferências racionalmente; existe uma função de preferência que tem como objetivo agrupar os múltiplos critérios num único critério de síntese. Tal abordagem considera a transitividade de preferências e indiferenças, mas exclui a incomparabilidade. As abordagens deste grupo vão desde o uso de médias ponderadas até o uso da Teoria da Utilidade Multiatributo (Gomes et al, 2002). Já os métodos não compensatórios (Escola Francesa ou Européia) consideram a incomparabilidade entre alternativas, não existe transitividade e/ou indiferenças. Dentre os métodos mais utilizados, destacam-se as famílias de métodos PROMETHEE e ELECTRE (GOMES et al, 2002).

O método PROMETHEE (Preference Method for Enrichment Evaluation) (Brans \& Vincke, 1985) procura estabelecer uma relação de sobreclassificação de valores, superação ou prevalência de alternativas em relação às demais. Os métodos de sobreclassificação favorecem ações balanceadas em detrimento de ações que são muito boas para determinado(s) critério(s) e muito ruins em outro(s). Segundo Almeida \& Costa (2002), esse método destaca-se por buscar envolver conceitos e parâmetros, os quais têm alguma interpretação física ou econômica, facilmente entendida pelo decisor; tendo sido descrito numa quantidade considerável de papers e aplicado com sucesso em vários problemas de diferentes naturezas.

A grande aplicabilidade do método deve-se a sua característica de ser um método de ordenação simples na concepção e ordenação, quando comparado a outros método de análise multicritério. É adequado a problemas onde existe um número finito de alternativas que estão sendo ordenadas, considerando vários critérios conflitantes; adaptando-se ao tipo de problema que se deseja abordar neste artigo: a priorização de projetos de TI/SI para serem desenvolvidos, considerando certo número de critérios, quem podem ser conflitantes. Para um critério j, temos $g_{j}(a)$ que representa o desempenho da alternativa "a" para esse critério, cada alternativa correspondendo a uma aplicação de SI. 
Um peso $p_{j}$, que representa o grau de importância relativa entre os critérios, deve ser atribuído para cada critério considerado. O PROMETHEE apresenta seis formas diferentes de o decisor representar suas preferências entre o desempenho das alternativas para cada critério $\left[g_{j}(a)-\right.$ $\mathrm{g}_{\mathrm{j}}(\mathrm{b})$ ], não necessariamente usando a mesma forma para todos os critérios. $\mathrm{O}$ decisor pode definir uma função $\mathrm{F}(\mathrm{a}, \mathrm{b})$ que assume valores entre 0 e 1, aumentando caso a diferença de desempenho ou a vantagem de uma alternativa em relação a outra aumenta, ou é igual a zero se o desempenho de uma alternativa for igual ou inferior ao da outra (ALMEIDA \& COSTA, 2002).

Com as intensidades de preferência já estabelecidas, calcula-se o grau de sobreclassificação $\pi(\mathrm{a}, \mathrm{b})$ para cada par de alternativas $(\mathrm{a}, \mathrm{b})$ :

$\pi(\mathrm{a}, \mathrm{b})=\frac{1}{P} \sum_{j=1}^{n} p_{j} F_{j}(a, b)$

onde

$\mathrm{P}=\sum_{j=1}^{n} p_{j}$

Em seguida, obtem-se o fluxo liquido de saída:

$\Phi+=\Sigma \pi(\mathrm{a}, \mathrm{b})$, representa a intensidade de preferência de a sobre todas as alternativas;

$\Phi-=\Sigma \pi(\mathrm{b}, \mathrm{a})$, representa a intensidade de preferência de todas alternativas sobre a;

$\Phi=\Phi+$ - $\Phi$-, chamado fluxo líquido, estabelece uma ordem completa entre as alternativas

Para cada projeto de TI atribui-se um escore, representado pelo fluxo líquido $\Phi$, obtido com a aplicação do PROMETHEE II. Ordenar os projetos segundo tal escore, porém, não é suficiente. Restrições financeiras, de precedência técnica e aspectos gerenciais devem ser levadas em conta na priorização destes projetos.

\section{Programação Inteira}

Programação inteira lida com problemas de otimização de uma função de várias variáveis sujeita a limitações do tipo equações e/ou inequações e sujeita a restrições em que uma ou mais variáveis devem assumir apenas valores inteiros. Em muitos modelos, variáveis inteiras são usadas para representar relações lógicas e por isso são limitadas a serem iguais a 0 ou 1 , caso que denominamos problema de programação inteira 0-1 (zero-um) (NEMHAUSER \& WOLSEY, 1999).

O problema aqui tratado é o de escolher um subconjunto dos projetos que maximize a soma de escores (representados pelos fluxos líquidos de cada projeto), dos projetos selecionados, sem exceder as limitações orçamentárias; além de obedecer certo equilíbrio entre as áreas da empresa que serão terão seus projetos desenvolvidos e restrições de precedência técnica. 
- N: conjunto de projetos;

- M: conjunto de áreas dos projetos;

- Custoi: custo do projeto ' $i$ ';

- Escorei: escore do projeto ' $i$ ';

- Areai: área ao qual o projeto 'i' está associado;

- Investminj: número mínimo de projetos que devem ser selecionados na área 'j';

- Investmaxj: número máximo de projetos que podem ser selecionados na área 'j';

- Recursos: recurso total disponível.

E, a seguinte variável de decisão:

$-\mathrm{Xi}$, em que $\mathrm{xi}_{\mathrm{i}}=1$, se o projeto i for selecionado ou $\mathrm{xi}=0$, caso contrário.

O modelo de programação matemática, baseado em Mavrotas et al (2006) e Ghasemzadeh et al (1999), para o problema é:

MAX $\sum_{i=1}^{n} \operatorname{escore}_{i} \times x_{i}$

s.a:

$\sum_{i=1}^{n}$ custo $_{i} \times x_{i} \leq$ recursos

$\sum_{\mathrm{i} \in A} x_{i}$ investMIN $_{A}$, para A = Área1, Área2,..., Área n;

$\sum_{i \in A} x_{i} \leq$ invest $M A X_{A}$, para $\mathrm{A}=$ Área1, Área2, ..., Área n;

$x_{i} \leq x_{p}$, onde $\mathrm{x}_{\mathrm{p}} \in \mathrm{P}_{\mathrm{i}}$, conjunto de projetos que são precedentes técnicos de $\mathrm{x}_{\mathrm{i}}$;

$x_{i} \in\{0,1\}, \forall i \in N$;

Ao final do modelo proposto, obtém-se os projetos de TI cujo desenvolvimento deve ser priorizado. Este trabalho descreve uma aplicação deste modelo numa empresa pública de fomento de informática, localizada na região Nordeste do Brasil. O nome da empresa será mentido em sigilo.

\section{Aplicação do modelo proposto}

A empresa de fomento de informática agrega sua experiência em tecnologia da informação, gerência de dados e recursos computacionais objetivando o atendimento das demandas formuladas pela Administração Pública Estadual. Dentro os serviços prestados relaciona-se: provedor de rede de teleinformática; provedor de acesso a serviços e informações e servidor computacional de serviços e informações. Tem como missão fomentar, coordenar, integrar, comercializar e executar soluções em tecnologia da informação e da comunicação, atender à demanda do mercado, 
prioritariamente da Administração Pública Estadual, apoiando o desenvolvimento do Estado, a melhoria contínua do serviço público, da qualidade de vida e organização da sociedade, criando parcerias entre o Estado, a iniciativa privada e os cidadãos, buscando a excelência da democratização da informação.

Suas diretrizes são:

- Promoção, intensificação e diversificação do uso da informática na Administração Pública Estadual;

- Disponibilização da tecnologia da informação para o atendimento direto ao cidadão;

- Integração e consolidação dos sistemas corporativos e estratégicos do Estado;

- Implantação e expansão da rede digital do Estado;

- Atualização da base tecnológica da empresa com a absorção de novas tecnologias.

Os Fatores Críticos de Sucesso identificados foram:

a) Nível de satisfação dos clientes

b) Potenciais áreas para novos negócios

c) Custos

Da metodologia de planejamento de sistemas de informação BSP (Almeida \& Ramos, 2003), resulta as aplicações que formarão o portfolio de SI, a seguir apresentados. Além destas aplicações, a Tabela 1 fornece o investimento necessário (em reais) e informa, se existir, a dependência técnica do projeto.

Tabela 1 - Aplicações de SI identificadas para a empresa

\begin{tabular}{|c|c|c|c|c|c|}
\hline 1 Informações & 2 Informação & 3 Suporte a & 4 Informações & 5 Automação & 6 Informações \\
\hline Transacionais de & Gerenciais de & Decisão no & Executivas de & de Escritório & Transacionais \\
\hline $\begin{array}{l}\text { Planejamento } \\
\text { (R\$32mil) }\end{array}$ & $\begin{array}{l}\text { Planejamento } \\
\text { (R } \$ 17 \text { mil) } \\
\quad \text { depende de } 1\end{array}$ & $\begin{array}{l}\text { Planejamento } \\
\text { (R\$60mil) }\end{array}$ & $\begin{array}{l}\text { Planejamento } \\
\text { (R\$70mil) }\end{array}$ & $\begin{array}{l}\text { Planejamento } \\
(\mathrm{R} \$ 30 \mathrm{mil})\end{array}$ & $\begin{array}{l}\text { de Pessoal } \\
\text { (R\$20mil) }\end{array}$ \\
\hline 7 Informação & 8 Suporte a & 9 Informações & 10 Automação & 11 Informações & 12 Informação \\
\hline Gerenciais de & Decisão em & Executivas de & de Escritório & Transacionais & Gerenciais de \\
\hline $\begin{array}{l}\text { Pessoal }(\mathrm{R} \$ 12 \mathrm{mil}) \\
\text { depende de } 6\end{array}$ & Pessoal(R\$40mil) & Pessoal(R\$35mil) & $\begin{array}{l}\text { de Pessoal } \\
(\mathrm{R} \$ 15 \mathrm{mil})\end{array}$ & $\begin{array}{l}\text { de Finanças } \\
\text { (R\$35mil) }\end{array}$ & $\begin{array}{l}\text { Finanças } \\
\text { (R\$11mil) } \\
\text { depende de } 11\end{array}$ \\
\hline 13 Suporte a & 14 Informações & 15 Informações & 16 Informação & 17 Suporte a & 18 Informações \\
\hline Decisão em & Executivas de & Transacionais de & Gerenciais de & Decisão no & Executivas de \\
\hline $\begin{array}{l}\text { Finanças } \\
\text { (R\$52mil) }\end{array}$ & $\begin{array}{l}\text { Finanças } \\
\text { (R\$60mil) }\end{array}$ & $\begin{array}{l}\text { Serviços } \\
\text { (R\$20mil) }\end{array}$ & $\begin{array}{l}\text { Serviços } \\
\text { (R\$15mil) } \\
\text { depende de } 15\end{array}$ & $\begin{array}{l}\text { Serviços } \\
\text { (R\$42mil) }\end{array}$ & $\begin{array}{l}\text { Serviços } \\
\text { (R\$40mil) }\end{array}$ \\
\hline $\begin{array}{l}19 \text { Automação } \\
\text { de Escritório } \\
\text { de Serviços } \\
\text { (R\$20 mil) }\end{array}$ & $\begin{array}{l}20 \text { Informações } \\
\text { Transacionais de } \\
\text { Operação } \\
\text { (R\$37mil) }\end{array}$ & $\begin{array}{l}21 \text { Informação } \\
\text { Gerenciais de } \\
\text { Operação } \\
\text { (R } \$ 17 \text { mil) } \\
\text { depende de } 20\end{array}$ & $\begin{array}{l}22 \text { Suporte a } \\
\text { Decisão na } \\
\text { Operação } \\
\text { (R\$40mil) }\end{array}$ & $\begin{array}{l}23 \text { Informações } \\
\text { Executivas de } \\
\text { Operação } \\
\text { (R\$40mil) }\end{array}$ & $\begin{array}{l}24 \text { Automação } \\
\text { de Escritório } \\
\text { de Operação } \\
\text { (R\$35mil) }\end{array}$ \\
\hline
\end{tabular}




\begin{tabular}{|c|c|c|c|c|c|}
\hline $\begin{array}{l}20 \text { Informações } \\
\text { Transacionais de } \\
\text { Operação } \\
\text { (R\$37mil) }\end{array}$ & $\begin{array}{l}21 \text { Informação } \\
\text { Gerenciais de } \\
\text { Operação } \\
\text { (R } \$ 17 \text { mil) } \\
\text { depende de } 20\end{array}$ & $\begin{array}{l}22 \text { Suporte a } \\
\text { Decisão na } \\
\text { Operação } \\
(\mathrm{R} \$ 40 \text { mil })\end{array}$ & $\begin{array}{l}23 \text { Informações } \\
\text { Executivas de } \\
\text { Operação } \\
(\mathrm{R} \$ 40 \mathrm{mil})\end{array}$ & $\begin{array}{l}24 \text { Automação } \\
\text { de Escritório } \\
\text { de Operação } \\
(\mathrm{R} \$ 35 \text { mil) }\end{array}$ & $\begin{array}{l}25 \text { Automa } \\
\text { ção da } \\
\text { Produção } \\
\text { na } \\
\text { Operação } \\
\text { (R\$50mil) }\end{array}$ \\
\hline $\begin{array}{l}26 \text { Informações } \\
\text { Transacionais de } \\
\text { Novas Tecnologias } \\
\text { (NT) (R \$25mil) }\end{array}$ & $\begin{array}{l}27 \text { Suporte a } \\
\text { Decisão em } \\
\text { NT (R\$70mil) }\end{array}$ & $\begin{array}{l}28 \text { Informações } \\
\text { Executivas de } \\
\text { NT (R\$65mil) }\end{array}$ & $\begin{array}{l}29 \text { Automação } \\
\text { de Escritório } \\
\text { de NT (R\$15mil) }\end{array}$ & $\begin{array}{l}29 \text { Automação } \\
\text { de Escritório } \\
\text { de NT(R\$15mil) }\end{array}$ & $\begin{array}{l}30 \text { Informações } \\
\text { Transacionais } \\
\text { De } \\
\text { Comercialização } \\
\text { (R\$20mil) }\end{array}$ \\
\hline $\begin{array}{l}30 \text { Informações } \\
\text { Transacionais de } \\
\text { Comercialização } \\
\text { (R\$20mil) }\end{array}$ & $\begin{array}{l}31 \text { Informação } \\
\text { Gerenciais de } \\
\text { Comercialização } \\
\text { (R\$14mil) } \\
\text { Depende de } 30\end{array}$ & $\begin{array}{l}32 \text { Suporte a } \\
\text { Decisão em } \\
\text { Comercialização } \\
(\mathrm{R} \$ 75 \mathrm{mil})\end{array}$ & $\begin{array}{l}33 \text { Informações } \\
\text { Executivas de } \\
\text { Comercialização } \\
\text { (R\$70mil) }\end{array}$ & $\begin{array}{l}34 \text { Automação } \\
\text { de Escritório } \\
\text { de } \\
\text { Comercialização } \\
(\mathrm{R} \$ 15 \mathrm{mil})\end{array}$ & \\
\hline
\end{tabular}

A partir dos Fatores Críticos de Sucesso, foram indicados os seguintes critérios (Tabela 2), utilizados na aplicação do método PROMETHEE II. Os potenciais projetos de SI são avaliados e ordenados de forma decrescente (Tabela 3).

Tabela 2 - Critérios utilizados na avaliação dos SI's

\begin{tabular}{lccc}
\hline Grupo de critério & Critério & Critério geral & Peso \\
\hline Estratégico & Qualidade de serviço $(\mathrm{c} 1)$ & pseudo-critério $(\mathrm{q}=0,04, \mathrm{p}=0,06)$ & 0,18 \\
& Novas tecnologias $(\mathrm{c} 2)$ & pseudo-critério $(\mathrm{q}=0,04, \mathrm{p}=0,06)$ & 0,06 \\
& Oportunidade de novos negócios $(\mathrm{c} 3)$ & quase-critério $(\mathrm{q}=0,03)$ & 0,18 \\
& Custo $(\mathrm{c} 4)$ & critério usual ou verdadeiro & 0,18 \\
Processo & Importância da automação $(\mathrm{p} 1)$ & critério usual ou verdadeiro & 0,20 \\
& Compromisso do usuário $(\mathrm{p} 2)$ & critério usual ou verdadeiro & 0,04 \\
Técnico & Oportunidade tecnológica $(\mathrm{t} 1)$ & critério usual ou verdadeiro & 0,10 \\
& Custo relativo da tecnologia $(\mathrm{t} 2)$ & critério usual ou verdadeiro & 0,06 \\
\hline
\end{tabular}

Tabela 3 - Fluxos (escores) dos SI's

\begin{tabular}{llll}
\hline Projetos & $\phi+$ & $\phi-$ & $\phi$ \\
\hline 3 & 21,02 & 0,67 & 20,35 \\
4 & 21,02 & 0,67 & 20,35 \\
15 & 18,89 & 3,4 & 15,49 \\
16 & 18,89 & 3,88 & 15,01 \\
20 & 19,98 & 5,04 & 14,94 \\
28 & 17,09 & 3,01 & 14,08 \\
18 & 17,02 & 3,96 & 13,06 \\
17 & 16,54 & 4,12 & 12,42 \\
21 & 16,87 & 5,68 & 11,19 \\
27 & 13,44 & 4,47 & 8,97 \\
34 & 8,56 & 0 & 8,56 \\
31 & 12 & 7,49 & 4,51 \\
33 & 11,06 & 6,74 & 4,32 \\
14 & 9,92 & 8,98 & 0,94 \\
12 & 9,56 & 8,83 & 0,73 \\
32 & 7,38 & 6,74 & 0,64 \\
30 & 8,98 & 8,82 & 0,16 \\
6 & 9,04 & 8,99 & 0,05 \\
25 & 9,06 & 10,43 & $-1,37$ \\
19 & 7,62 & 10,68 & $-3,06$
\end{tabular}




\begin{tabular}{llll}
7 & 7,76 & 11,08 & $-3,32$ \\
22 & 7,94 & 11,55 & $-3,61$ \\
11 & 6,12 & 12,72 & $-6,6$ \\
10 & 5,74 & 13,64 & $-7,9$ \\
23 & 5,06 & 13,95 & $-8,89$ \\
26 & 5 & 14,27 & $-9,27$ \\
13 & 5,1 & 15,66 & $-10,56$ \\
8 & 4,4 & 16,08 & $-11,68$ \\
9 & 4,4 & 16,08 & $-11,68$ \\
5 & 3,8 & 16,57 & $-12,77$ \\
29 & 3,2 & 17,71 & $-14,51$ \\
2 & 2,06 & 17,8 & $-15,74$ \\
24 & 2,28 & 18,63 & $-16,35$ \\
1 & 0,46 & 20,36 & $-19,9$ \\
\hline
\end{tabular}

Para cada projeto é identificada a área ao qual pertence, o orçamento previsto para sua realização e as possíveis relações de precedência técnica entre os projetos. Em seguida, o modelo de programação inteira é construído, considerando-se o recurso financeiro total disponível - R\$ 400.000,00 - e a quantidade máxima e mínima de projetos por área em função da disponibilidade de cada área (Tabela 4).

Tabela 4 - Números máximo e mínimo de projetos que podem ser implementados por área

\begin{tabular}{lccc}
\hline Área & Máximo & Mínimo & 1 \\
\hline Gerencia de núcleo de apoio a gestão & 3 & 2 \\
Gerencia administrativa e financeira & 4 & 3 \\
Gerência de Infra-estrutura e Serviços & 6 & 2 \\
Gerência Executiva de Tecnologia da & & 1 \\
Informação e Comunicação & 6 & 1 \\
Gerência de Desenvolvimento & 3 & 1 \\
Gerência de Relacionamento & 3 & \\
\hline
\end{tabular}

Os dados acima permitem a aplicação do modelo proposto neste artigo, definindo as aplicações de SI da empresa analisada que devem ser priorizadas. A etapa de programação inteira foi realizada com a utilização do software LINGO, em sua versão para estudantes, de forma integrada com o Microsoft EXCEL. O resultado do modelo está descrito a seguir. 
Tabela 5 - Aplicações de SI's que devem ser priorizadas pela empresa

\begin{tabular}{cl}
\hline Projeto & \multicolumn{1}{c}{ Identificação } \\
\hline 3 & Suporte a Decisão no Planejamento \\
6 & Informações Transacionais de Pessoal \\
7 & Informações Gerenciais de Pessoal \\
10 & Automação de Escritório de Pessoal \\
11 & Informações Transacionais de Finanças \\
12 & Informações Gerenciais de Finanças \\
14 & Informações Executivas de Finanças \\
15 & Informações Transacionais de Serviços \\
16 & Informações Gerenciais de Serviços \\
19 & Automação de Escritório de Serviços \\
20 & Informações Transacionais de Operação \\
21 & Informações Gerenciais de Operação \\
26 & Informações Transacionais de Novas Tecnologias \\
30 & Informações Transacionais de Comercialização \\
31 & Informação Gerenciais de Comercialização \\
34 & Automação de Escritório de Comercialização \\
\hline
\end{tabular}

O modelo priorizou dezesseis projetos dentre os trinta e quatro identificados pela Metodologia BSP. A escolha levou em consideração a restrição de orçamento, a intensidade de preferências pelos projetos (escores resultantes do método PROMETHEE II), o requisito de precedência técnica e a capacidade de cada área da empresa se envolver com os projetos. O modelo estabelece então um equiíbrio de projetos entre as áreas, não concentrando todos os projetos e, consequentemente, os recursos em uma única área da empresa.

\section{Conclusões}

Este artigo discutiu a problemática de investimentos em tecnologia da informação (TI), sobretudo quanto à priorização de potenciais aplicações de TI/SI, que compõem o portfolio de investimentos deste setor da empresa.

A resolução do problema citado requer a estruturação de um modelo de decisão apropriado às características do segmento analisado (TI/SI), com a inclusão de diversos fatores que devem estar presentes na avaliação de um investimento na área. Além disso, há necessidade de incorporar restrições orçamentárias, técnicas, gerenciais, etc. $\mathrm{O}$ modelo proposto utilizou o método PROMETHEE II (Brans \& Vincke, 1985) e, posteriormente, incorporou diferentes restrições do problema utlizando-se de programação inteira 0-1. Uma aplicação do modelo foi apresentada, envolvendo uma empresa estadual de fomento de TI, localizada no Nordeste brasileiro, e como resultado foram estabelecidos os projetos que devem ser priorizados.

Para solução do problema, foi utilizado o software LINGO, em sua versão para estudantes. Entre as dezesseis aplicações de SI a serem priorizadas, destaca-se a grande quantidade de sistemas transacionais e gerenciais, que baseiam toda a estrutura de TI das empresas, sobretudo nas áreas 
mais operacionais: operações e comércio. Além disso, observa-se a escolha de sistemas executivos e de apoio a decisão em setores de importância estratégica: finanças e planejamento.

Pesquisas que envolvam a introdução de novas restrições, como fatores de risco associados aos investimentos em TI/SI, podem complementar o presente artigo aprimorando o modelo proposto.

\begin{abstract}
Actuate in an environment of competition requires companies the efficient use of its financial resources in order to bring competitive advantage, driving the business forward. To achieve the potential benefits of information technology (IT) and information systems (IS) is paramount efficient management of these resources, especially in decisions to invest in information technology. TI can not be judged solely by financial gain but also by other contributions it brings to the various aspects of the business, which characterized the problem of investment in IT / IS as a multicriteria decision problem. In this work, we proposed a decision model based on the method PROMETHEE V, a combination of the multicriteria decision method PROMETHEE II and integer programming 0-1, involving different types of attributes and restrictions on the problem.
\end{abstract}

Key-words: investment selection, information technology, decision-aid.

\title{
Referências
}

AHITUV, N.; NEUMANN, S. Principles of information systems for management. Vm. C. Brown Company Publishing. 1983.

ALBUQUERQUE, R. M. de; COSTA A. P. C. S. Apoio multicritério a decisão e programação inteira 0-1 aplicados ao problema de priorização de sistemas de informação. Anais do XXVI ENEGEP - Encontro Nacional de Engenharia de Produção, Fortaleza, 2006

ALMEIDA, A.T. \& COSTA, A. P. C. S. Modelo de decisão para priorização no Planejamento de Sistemas de Informação. Gestão \& Produção, v. 9, n.2, p. 201-214, 2002.

ALMEIDA, A.T. \& COSTA, A. P. C. S. Aplicações com métodos multicritério de apoio a decisão. Recife: Ed. Universitária UFPE, 2003.

BRANS, J. P.; VINCKE, P.H. A preference ranking organization method, the PROMETHEE method for MCDM. Mgmt. Sci., v.31, p647-656, 1985.

cross ${ }^{\text {ref }}$

BRANS, J. P.; MARESCHAL, B. PROMETHEE V: MCDM problems with segmentation constraints. INFOR, v. 30, n. 2, p. 85-86, 1992.

GHASEMZADEH, F.; ARCHER, N. \& IYOGUN, P. A zero-one model for project portfolio selection and scheduling. Journal of the operational Research Society, 50; 745-755, 1999.

cross ${ }^{\text {ref }}$

GOMES, L.F.A.M.; GOMES, C.F.S. \& ALMEIDA, A.T. Tomada de Decisão Gerencial: Enfoque Multicritério. Editora Atlas, Rio de Janeiro, 2002.

LOPES, Y. G.; COSTA A. P. C. S. O planejamento e a gestão da tecnologia da informação: um estudo nas empresas pernambucanas. Anais do XXVI ENEGEP - Encontro Nacional de Engenharia de Produção, Fortaleza, 2006. 
MAVRotas, G.; DiAKOUlAKI, D. \& CALOGHIROU, Y. Project priorization under policy restrictions. A combination of MDCA with 0-1 programming. European Journal of Operational Research, 171:296-308, 2006.

cross ${ }^{\text {ref }}$

MELLO,J. C. C. B. S. de; GOMES, E.G.; GOMES, L. F. A. M. ; NETO, L.B.; MEZA, L.A. Avaliação do tamanho de aeroportos portugueses com relações multicritério de relação. Pesquisa Operacional, v.25, n.3, p.313-330, 2005

NEWHAUSER, G. L. \& WOLSWY, L. A. Integer and combinatorial optimization. Wiley - Interscience, Estados Unidos, 1999.

SCHWARZ, A. \& HIRSCHHEIM, R. An extended platform logic perspective of IT governance: managing perceptions and activities of IT. Journal of strategic Informations Systems, Vol.12, p. 129-166, 2003.

VINCKE, P. Multicriteria decision-aid. Londres: Jonh Wiley \& Sons, 1992.

\section{Dados dos autores:}

Nome completo: Yuri Gama Lopes

Filiação institucional: UFPE

Departamento: Engenharia de Produção

Função: Estudante de graduação

Endereço completo: Rua Antônio Passos, número 334, apt $^{\mathrm{o}} 102$ CEP 51030-040, Boa Viagem, Recife - PE, Brasil

Telefones para contato: (81) 2126-8728 Ramal 29

E-mail: yurilopes@ufpe.br

Nome completo: Ana Paula Cabral Seixas Costa

Filiação institucional: UFPE

Departamento: Engenharia de Produção

Função: Professora - Dedicação exclusiva

Endereço completo: Universidade federal de Pernambuco, Centro de Tecnologia e Geociências, Bloco Administrativo - $5^{\circ}$ andar, Av. Acadêmico Hélio Ramos, s/n - Cidade Universitária , Recife PE CEP: 50.740-530, Recife - PE, Brasil

Telefones para contato: (81)21268728

E-mail: apcabral@ufpe.br 